\title{
Analysis and Experimental Study on Dynamic Behavior of Stepper-Actuated Dual-Axis Data Transmission Antenna
}

\author{
Zhaomingyue Zheng $\mathbb{D},{ }^{1}$ Wei Cheng, ${ }^{1}$ Ming Li $\mathbb{D},{ }^{1}$ Guangyuan Wang $\mathbb{D},{ }^{2}$ and Limeng Tan ${ }^{3}$ \\ ${ }^{1}$ School of Aeronautic Science and Engineering, Beihang University, Beijing 100191, China \\ ${ }^{2}$ China Academy of Space Technology, Beijing, China \\ ${ }^{3}$ Beijing Institute of Spacecraft System Engineering, Beijing, China
}

Correspondence should be addressed to Ming Li; li_ming@buaa.edu.cn and Guangyuan Wang; zhuichilun@126.com

Received 17 December 2020; Revised 4 March 2021; Accepted 25 March 2021; Published 15 April 2021

Academic Editor: Mohammad Tawfik

Copyright (C) 2021 Zhaomingyue Zheng et al. This is an open access article distributed under the Creative Commons Attribution License, which permits unrestricted use, distribution, and reproduction in any medium, provided the original work is properly cited.

\begin{abstract}
The satellite-borne data transmission antenna is the main disturbing source of low-frequency microvibration of spacecraft, which immensely affects the image quality of remote sensing satellite. In this paper, the dynamic characteristics of flexible load driven by stepping motor on flexible boundary are studied. The dynamic equation of the stepping motor driven by current subdivision is simplified by linearization method. The dynamic model of flexible load driven by stepping motors on flexible boundary is established by using the Dynamic Substructure Method, and the analytical expression of microvibration of the data transmission antenna is given. The coupling relationship between the stepping motors and the flexible structure is analyzed by modal coordinate transformation. The microvibration model is verified by simulation and experiment, and the main causes and coupling factors of microvibration are explained. The results show that the model can accurately predict the microvibration of the satellite antenna and can be applied to the microvibration prediction in orbit. The reasonable selection of the working velocity of the stepping motors can effectively reduce the microvibration, which provides the basis for the design of the antenna control system.
\end{abstract}

\section{Introduction}

In order to improve the imaging quality of high-resolution remote sensing satellite, the stability requirements of remote sensing satellite are constantly raised [1]. But there are control moment gyroscope, momentum wheel, solar array and solar array drive assembly, data transmission antenna, and other moving parts on the satellite [2-5]. When the satellite is in orbit, the moving parts will cause microvibration. This phenomenon not only affects the resolution and pointing accuracy of payloads such as cameras but also affects the attitude control of satellites [6]. The spaceborne data transmission antenna plays an important role in spacecraft data relay and intersatellite link tasks, but it is also the main source of low-frequency microvibration [7-9].

Hybrid two-phase stepping motor is widely used in lowspeed rotation of spacecraft moving parts due to its simple structure, high positioning accuracy, and easy open-loop control circuit $[10,11]$. At present, Subdivision Drive (SDD) is widely used, which can improve the resolution of the motor [12] and the stability of operation [13] by subdividing the current change into multiple microelectric pulses. The satellite-borne data transmission antenna is driven by two vertically arranged stepper motors to rotate the reflector [14]. The reflector is a flexible structure with large size, which is connected with the support arm through the driving mechanism. In order to improve the pointing range of the antenna and avoid the structural interference between the reflector and the satellite body, the support arm of the antenna is designed longer. This makes the support arm a flexible structure. When the antenna is working, the electric pulse signal of the stepping motor and the harmonic drive of the meshing teeth of the deceleration mechanism will cause microdisturbance $[15,16]$. The vibration may be coupled with the flexible load reflector and the flexible boundary support arm, which will further amplify the vibration. This 
kind of microdisturbance may cause structural vibration of the flexible load reflector and the flexible boundary support arm, which will react on the stepping motors. Furthermore, the coupling vibration not only affects the performance of the payload but also affects the pointing accuracy of the antenna itself. Therefore, the microvibration phenomenon of the data transmission antenna can be summarized as the coupling vibration problem of the flexible load driven by stepping motors on the flexible boundary. The modeling and analysis of this problem is more complex.

Most researchers take the pointing accuracy of data transmission antenna as the design goal [9] and model from the stepping motor and the control method. Liu et al. analyzed the influencing factors of the pointing accuracy of the data transmission antenna from the aspects of pointing algorithm, pointing execution, ground station pointing, and atmospheric transmission [17]. Zhou et al. established the drive mechanism model of the data transmission tracking antenna by considering the nonlinearity of the harmonic gear and the dynamic characteristics of the driving circuit [18]. Although this kind of method can be used to analyze the pointing accuracy of the antenna, it cannot be used to predict the torque of the microvibration of the antenna. Some researchers have established the dynamic model of the data transmission antenna from the perspective of the flexible structure. Song established the dynamic model of the data transmission antenna through the Finite Element Method and calculated and analyzed the vibration characteristics [19]. Cao et al. established the flexible coupling dynamic equation of the data transmission antenna by using the loading mode synthesis hybrid coordinate method [20]. Their research does not consider the coupling effect between the stepping motors and the flexible structure and could not accurately explain the microvibration phenomenon of the antenna.

In the research of the coupling vibration between stepping motor and structure, Yang et al. verified the existence of electromagnetic stiffness of the stepping motor through experiments and simulation [21]. According to the principle of stepping motor, Liu simplified the load to rigidity and established the general analytical model and simplified model of microvibration of the data transmission antenna [22]. Chen et al. established the vibration equation of the stepping motor by simplifying and linearizing the electromagnetic torque of the stepping motor and carried out simulation analysis with a two degree of freedom flexible system [23]. According to the Lagrange Energy Method, Sattar et al. established the dynamic model of kinematics and microvibration coupling between the stepping motor and the rigid load [24]. Other researchers have studied the microvibration phenomenon of the antenna through experiments, but have not discussed the cause and coupling principle in depth. Wu et al. proposed a semiempirical and semianalytical microvibration model of the data transmission antenna by considering the stepping motor and the structure modes of antenna [15]. Hyun-Ung Oh et al. tested the microvibration of a data transmission antenna using a force measuring platform manufactured by KISTLER and designed a pseudoelastic shape memory alloy-compressed mesh washer isolator to suppress the microvibration. But they did not reveal the coupling relationship between the stepper motor and the antenna structure [16]. Therefore, it is necessary to study the coupling vibration of the flexible load driven by stepping motors on the flexible boundary by mathematical modeling, at the same time verify and analyze the mechanism and influencing factors of microvibration of data transmission antenna through experiments.

By establishing the mathematical model of the flexible load driven by stepping motors on the flexible boundary to analyze the microvibration of the antenna not only can the electromechanical coupling relationship be revealed in essence but also has strong application value. Firstly, the microvibration data obtained from experiment and simulation can be used as design reference index of the antenna. By optimizing the control law and parameters, the microvibration of the antenna can be reduced from the source. Secondly, the coupling relationship of the system is analyzed, which can provide theoretical basis for vibration reduction and isolation design. Finally, in addition to the data transmission antenna, there are many flexible structures and moving parts in the satellite. The methods used in this paper can also provide reference for solving the same kind of coupling vibration problems.

This paper studies the dynamic model of the flexible load driven by stepping motors on the flexible boundary. The dynamic equation of stepping motor is simplified by linearization method. The dynamic model of the flexible load driven by stepping motors on the flexible boundary is established by using the Dynamic Substructure Method, and the analytical expression of the microvibration of the data transmission antenna is given. The coupling relationship of the system is analyzed by the coordinate change method. Finally, the method is verified by simulation and experiment.

\section{Microvibration Model of the Dual-Axis Data Transmission Antenna}

2.1. Simplification of Dynamic Equation of Stepping Motor Driven by Current Subdivision. The driving model of twophase hybrid stepping motor is:

$$
\left\{\begin{array}{l}
\boldsymbol{\Phi}=\mathbf{L I}, \\
T_{e}=\frac{1}{2} \mathbf{I}^{\mathrm{T}} \frac{\partial \mathbf{L}}{\partial \theta} \mathbf{I},
\end{array}\right.
$$

in which $\boldsymbol{\Phi}$ is the flux vector, $\mathbf{I}$ is the current vector, and $\mathbf{L}$ is the inductance matrix. The inductance matrix is symmetrical and ignores the higher-order harmonic components of periodic magnetic derivative function. The inductance matrix can be expressed as:

$\mathbf{L}=\left(\begin{array}{ccc}L_{0} & 0 & L_{m 0}+L_{m 1} \cos (z \theta) \\ 0 & L_{0} & L_{m 0}+L_{m 1} \sin (z \theta) \\ L_{m 0}+L_{m 1} \cos (z \theta) & L_{m 0}+L_{m 1} \sin (z \theta) & L_{n 0}+L_{n 1} \cos (4 z \theta)\end{array}\right)$,

in which $z$ is the number of teeth of the rotor. In order to make the stepper motor run smoothly, the driving current 
of the stepper motor is usually subdivided step by step, so the current vector I can be expressed as:

$$
\mathbf{I}=\left[\begin{array}{c}
I_{A} \\
I_{B} \\
I_{f}
\end{array}\right]=\left[\begin{array}{c}
I_{m} \cos (\gamma i) \\
I_{m} \sin (\gamma i) \\
I_{f}
\end{array}\right], \quad i=1,2,3, \cdots,
$$

in which $I_{A}$ and $I_{B}$ are two-phase winding current of $A$ and $B$, $I_{f}$ is equivalent excitation current of permanent magnet, $I_{m}$ is magnitude of current subdivision of two-phase winding, $\gamma$ is step angle of current subdivision, and $i$ is operation step.

The dynamic model of the two-phase hybrid stepping motor is:

$$
J_{0} \frac{\mathrm{d}^{2} \theta}{\mathrm{d} t^{2}}+C_{0} \frac{\mathrm{d} \theta}{\mathrm{d} t}=T_{e}-T_{l}
$$

in which $\theta$ is the rotor angle, $J_{0}$ and $C_{0}$ are the motor rotor inertia and the viscous damping coefficient inside the motor, and $T_{e}$ and $T_{l}$ are the electromagnetic torque and the load torque. According to Equations (1) to (3), the electromagnetic torque can be reduced to:

$$
\begin{aligned}
T_{e} & =\frac{1}{2} \mathbf{I}^{\mathrm{T}} \frac{\partial \mathbf{L}}{\partial \theta} \mathbf{I}=K_{m}\left[I_{B} \cos (z \theta)-I_{A} \sin (z \theta)\right]-K_{d} \cos (4 z \theta) \\
& =K_{m} I_{m} \sin \left[z\left(\frac{\gamma i}{z}-\theta\right)\right]-K_{d} \cos (4 z \theta),
\end{aligned}
$$

in which $K_{m}=z I_{f} L_{m 1}$ is the electromagnetic torque coefficient and $K_{d}=2 z I_{f}^{2} L_{n 1}$ is the positioning torque coefficient. Since the positioning torque coefficient is far less than the electromagnetic torque coefficient, the positioning torque $K_{d} \cos (4 z \theta)$ is ignored. $\gamma i / z$ is the theoretical equilibrium position of the motor rotor after the occurrence of the $i$ th electrical pulse signal, and $\theta$ is the angular displacement of the motor rotor during this period. In order to avoid the stepping motor out of step, the difference between the actual angular displacement of the motor rotor and the theoretical balance position should be kept between a mechanical step angle, that is, the value range of the difference between the two $(\gamma i / z)-\theta$ is as follows:

$$
-\gamma_{z} \leq \frac{\gamma i}{z}-\theta \leq \gamma_{z}, \gamma_{z}=\frac{\gamma}{z}=\frac{2 \pi}{z p n}
$$

in which $\gamma_{z}$ represents the mechanical step angle of the stepper motor and $p$ and $n$ are the running beats and the subdivisions number of the stepper motor. The working state of the data transmission antenna is low-speed operation. In order to make the data transmission antenna have high pointing accuracy, the mechanical step angle of the stepper motor is a small value, so $z((\gamma i / z)-\theta)$ is also a smaller angle value. Equation (5) can be linearized, that is, $\sin [z((\gamma i / z)-$

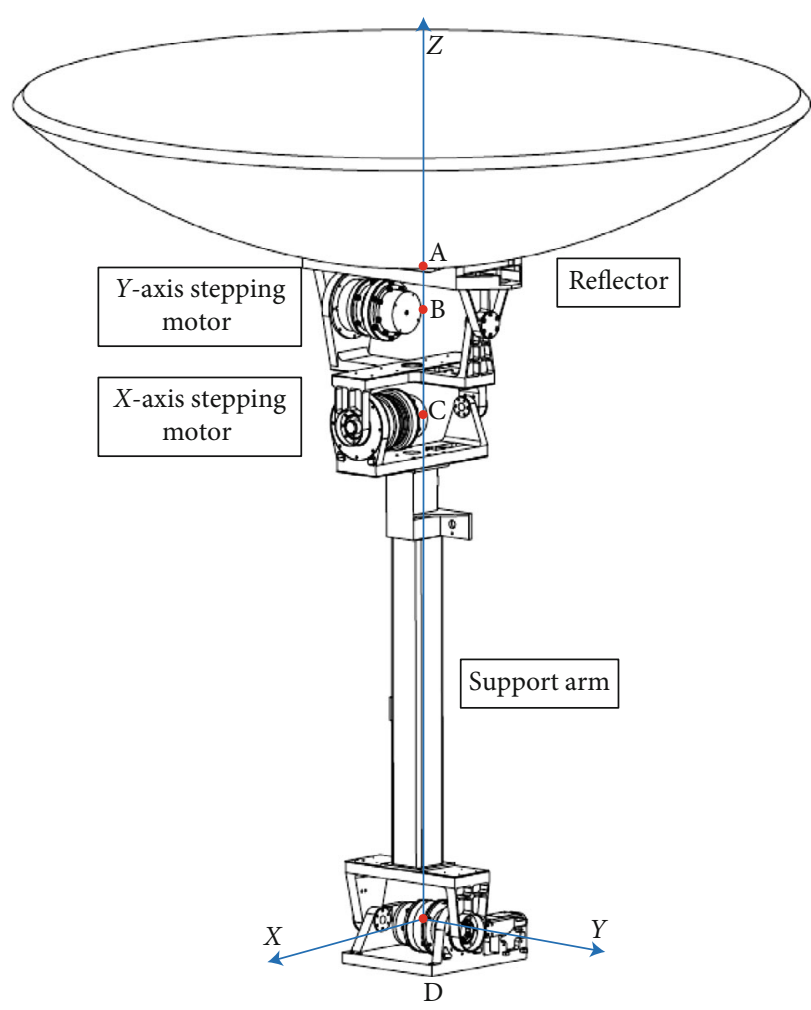

FIgURE 1: Structure diagram of dual-axis data transmission antenna.

$\theta)] \approx z((\gamma i / z)-\theta)$; the electromagnetic torque can be further simplified as follows:

$$
T_{e}=K_{m} I_{m} \gamma i-K_{m} I_{m} z \theta .
$$

Therefore, the dynamic equation of stepper motor driven by current subdivision can be converted into:

$$
J_{0} \frac{\mathrm{d}^{2} \theta}{\mathrm{d} t^{2}}+C_{0} \frac{\mathrm{d} \theta}{\mathrm{d} t}+K_{0} \theta=T_{e x}-T_{l},
$$

in which $K_{0}=K_{m} I_{m} z$ is the equivalent electromagnetic stiffness of the stepping motor. $T_{e x}=K_{m} I_{m} \gamma i$ is the step excitation component of electromagnetic torque, which is independent of the rotor angular displacement. Therefore, the stepping motor driven by current subdivision can be equivalent to an electromagnetic spring-damping system, which provides a basis for further analysis of the coupling vibration between the stepping motors and the flexible load and the flexible support arm.

2.2. Dynamic Model of the Dual-Axis Data Transmission Antenna. The dual-axis data transmission antenna consists of a support arm, an $x$-axis stepping motor, a $y$-axis stepping motor, and a reflector, in which the support arm and the reflector are flexible structures. Figure 1 shows the structure of a two-axis data transmission antenna. When the Finite Element Method is used to model the flexible structure, the degree of freedom of the structure is high, the amount of iteration calculation is large, and the efficiency is low. Therefore, the Dynamic Substructure Method is used to condensate the 


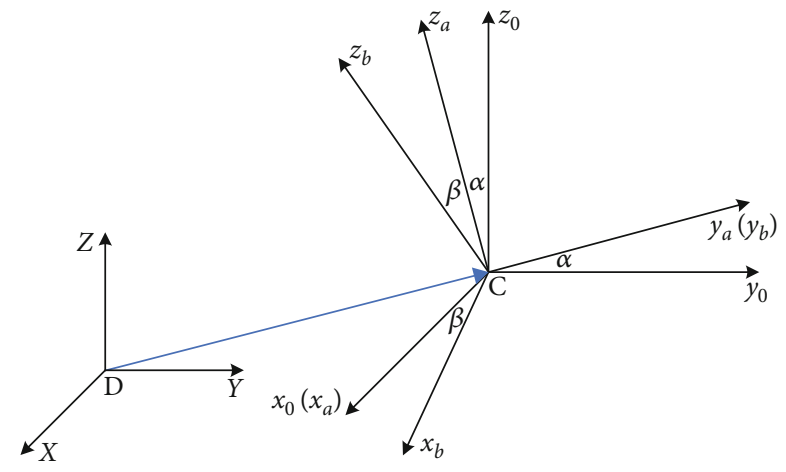

Figure 2: Coordinate system of dual-axis data transmission antenna.

dynamic model of flexible structure and convert the highdegree-of-freedom physical coordinates into low-degree-offreedom mode coordinates, which improves the computational efficiency. The microvibration of the antenna is mainly concentrated in the low-frequency band, so ignoring the influence of higher modes has little influence on the calculation accuracy.

Figure 2 shows the coordinate system of the dual-axis data transmission antenna. $X Y Z$ is defined as the inertial coordinate system, $x_{0} y_{0} z_{0}$ is the fixed coordinate system with the origin at point $\mathrm{C}, x_{a} y_{a} z_{a}$ is the intermediate coordinate system, and $x_{b} y_{b} z_{b}$ is the reflector coordinate system. $\alpha$ is defined as the rotation angular displacement of the $x$-axis motor, and $\beta$ is the rotation angular displacement of the $y$-axis motor. First, the $y$-axis motor and the reflector rotate $\alpha$ around the $x_{0}$-axis to the intermediate coordinate system, and then, the reflector rotates $\beta$ around the $y_{a}$-axis. The transformation relationship of coordinate system is as follows:

$$
\begin{aligned}
{\left[\begin{array}{l}
i_{0} \\
j_{0} \\
k_{0}
\end{array}\right] } & =\mathbf{R}\left(x_{0}, \alpha\right)\left[\begin{array}{l}
i_{a} \\
j_{a} \\
k_{a}
\end{array}\right]=\mathbf{R}\left(x_{0}, \alpha\right) \mathbf{R}\left(y_{a}, \beta\right)\left[\begin{array}{l}
i_{b} \\
j_{b} \\
k_{b}
\end{array}\right], \\
\mathbf{R}\left(x_{0}, \alpha\right) & =\left(\begin{array}{ccc}
1 & 0 & 0 \\
0 & \cos \alpha & \sin \alpha \\
0 & -\sin \alpha & \cos \alpha
\end{array}\right), \\
\mathbf{R}\left(y_{a}, \beta\right) & =\left(\begin{array}{ccc}
\cos \beta & 0 & -\sin \beta \\
0 & 1 & 0 \\
-\sin \beta & 0 & \cos \beta
\end{array}\right)
\end{aligned}
$$

in which $i_{0}, j_{0}$, and $k_{0}$ are the unit vectors of the fixed coordinate system; $i_{a}, j_{a}$, and $k_{a}$ are the unit vectors of the intermediate coordinate system; $i_{b}, j_{b}$, and $k_{b}$ are the unit vectors of the coordinate system; and $\mathbf{R}\left(x_{0}, \alpha\right)$ and $\mathbf{R}\left(y_{a}, \beta\right)$ are the coordinate transformation matrices.
The data transmission antenna is divided into four substructures, which are the reflector, the $y$-axis stepping motor, the $x$-axis stepping motor, and the support arm in turn. According to the Dynamic Substructure Method, the degrees of freedom of the substructure are divided into internal degrees of freedom and interface degrees of freedom. The dynamic equation of substructure 1 in its local coordinate system is:

$$
\begin{gathered}
{\left[\begin{array}{cc}
\mathbf{M}_{u u}^{b} & \mathbf{M}_{u A}^{b} \\
\mathbf{M}_{A u}^{b} & \mathbf{M}_{A A}^{b}
\end{array}\right]\left[\begin{array}{c}
\ddot{\mathbf{X}}_{u}^{b} \\
\ddot{\mathbf{X}}_{A}^{b}
\end{array}\right]+\left[\begin{array}{ll}
\mathbf{C}_{u u}^{b} & \mathbf{C}_{u A}^{b} \\
\mathbf{C}_{A u}^{b} & \mathbf{C}_{A A}^{b}
\end{array}\right]\left[\begin{array}{c}
\dot{\mathbf{X}}_{u}^{b} \\
\dot{\mathbf{X}}_{A}^{b}
\end{array}\right]} \\
+\left[\begin{array}{cc}
\mathbf{K}_{u u}^{b} & \mathbf{K}_{u A}^{b} \\
\mathbf{K}_{A u}^{b} & \mathbf{K}_{A A}^{b}
\end{array}\right]\left[\begin{array}{l}
\mathbf{X}_{u}^{b} \\
\mathbf{X}_{A}^{b}
\end{array}\right]=\left[\begin{array}{l}
\mathbf{F}_{u}^{b} \\
\mathbf{F}_{A}^{b}
\end{array}\right],
\end{gathered}
$$

in which superscript $b$ represents substructure 1 in local coordinate system, subscript $u$ represents internal degrees of freedom, and subscript $A$ represents interface degrees of freedom of interface node $\mathrm{A}$, and $\mathbf{M}^{b}=\left[\begin{array}{ll}\mathbf{M}_{u u}^{b} & \mathbf{M}_{u A}^{b} \\ \mathbf{M}_{A u}^{b} & \mathbf{M}_{A A}^{b}\end{array}\right]$, $\mathbf{C}^{b}=\left[\begin{array}{ll}\mathbf{C}_{u u}^{b} & \mathbf{C}_{u A}^{b} \\ \mathbf{C}_{A u}^{b} & \mathbf{C}_{A A}^{b}\end{array}\right]$, and $\mathbf{K}^{b}=\left[\begin{array}{ll}\mathbf{K}_{u u}^{b} & \mathbf{K}_{u A}^{b} \\ \mathbf{K}_{A u}^{b} & \mathbf{K}_{A A}^{b}\end{array}\right]$ are the mass matrix, damping matrix, and stiffness matrix of substructure 1 in its local coordinate system, respectively. With the Rayleigh damping model, the damping matrix is:

$$
\mathbf{C}^{b}=C_{1}^{b} \mathbf{M}^{b}+C_{2}^{b} \mathbf{K}^{b}
$$

in which $C_{1}^{b}$ and $C_{2}^{b}$ are Rayleigh damping coefficients of substructure 1 and $\mathbf{X}^{b}=\left[\begin{array}{l}\mathbf{X}_{u}^{b} \\ \mathbf{X}_{A}^{b}\end{array}\right]$ and $\mathbf{F}^{b}=\left[\begin{array}{c}\mathbf{F}_{u}^{b} \\ \mathbf{F}_{A}^{b}\end{array}\right]$ are the displacement and force column vectors. According to formula (9), the transformation relationship between the displacement vectors of the reflector in the local coordinate system $\mathbf{X}^{b}$ and the displacement vectors in the global coordinate system $\mathbf{X}^{1}$ is obtained as follows:

$$
\begin{aligned}
& \mathbf{X}^{1}=\mathbf{R}_{\alpha} \mathbf{R}_{\beta} \mathbf{X}^{b}, \\
& \mathbf{R}_{\alpha}=\operatorname{diag}\left[\mathbf{R}\left(x_{0}, \alpha\right)\right], \\
& \mathbf{R}_{\beta}=\operatorname{diag}\left[\mathbf{R}\left(y_{a}, \beta\right)\right], \\
& \mathbf{X}^{b}=\left[\begin{array}{l}
\mathbf{X}_{u}^{b} \\
\mathbf{X}_{A}^{b}
\end{array}\right] .
\end{aligned}
$$

According to the fixed interface mode synthesis method, the hypothetical mode set of substructure is 
composed of the main and constraint modes, and the solution of the main and constraint modes of substructure 1 is:

$$
\begin{array}{r}
\boldsymbol{\varphi}_{l}^{b}=\left[\begin{array}{c}
\boldsymbol{\varphi}_{u l}^{b} \\
\mathbf{0}_{A l}
\end{array}\right], \\
\left(\mathbf{K}_{u u}^{b}-\omega^{2} \mathbf{M}_{u u}^{b}\right) \boldsymbol{\varphi}_{u l}^{b}=0, \\
\boldsymbol{\varphi}_{A}^{b}=\left[\begin{array}{c}
\boldsymbol{\varphi}_{u A}^{b} \\
\mathbf{I}_{A A}
\end{array}\right],\left[\begin{array}{ll}
\mathbf{K}_{u u}^{b} & \mathbf{K}_{u A}^{b} \\
\mathbf{K}_{A u}^{b} & \mathbf{K}_{A A}^{b}
\end{array}\right]\left[\begin{array}{c}
\boldsymbol{\varphi}_{u A}^{b} \\
\mathbf{I}_{A A}
\end{array}\right]=\left[\begin{array}{c}
\mathbf{0}_{u A} \\
\mathbf{R}_{A A}
\end{array}\right],
\end{array}
$$

in which subscript $l$ represents the order of the main modes retained by substructure 1, and the first line of Equation (14) is expanded to obtain:

$$
\boldsymbol{\varphi}_{u A}^{b}=-\left(\mathbf{K}_{u u}^{b}\right)^{-1} \mathbf{K}_{u A}^{b}
$$

Therefore, the hypothetical modal set of substructure 1 in its local coordinate system is:

$$
\boldsymbol{\varphi}^{b}=\left[\boldsymbol{\varphi}_{l}^{b} \boldsymbol{\varphi}_{A}^{b}\right]=\left[\begin{array}{cc}
\boldsymbol{\varphi}_{u l}^{b} & \boldsymbol{\varphi}_{u A}^{b} \\
\mathbf{0}_{A l} & \mathbf{I}_{A A}
\end{array}\right]
$$

Substructures 2 and 3 are single-degree-of-freedom vibration systems in the free interface, and only one degree of torsional freedom exists. According to Equation (8), the dynamic equation of the stepping motors can be written as follows:

$$
\begin{aligned}
& M^{2} \frac{\mathrm{d}^{2} \beta}{\mathrm{d} t^{2}}+C^{2} \frac{\mathrm{d} \beta}{\mathrm{d} t}+K^{2} \beta=F^{2} \\
& M^{3} \frac{\mathrm{d}^{2} \alpha}{\mathrm{d} t^{2}}+C^{3} \frac{\mathrm{d} \alpha}{\mathrm{d} t}+K^{3} \alpha=F^{3}
\end{aligned}
$$

In which superscripts 2 and 3 represent substructures 2 and 3 , respectively, and each physical quantity has the meaning of:

$$
\begin{aligned}
& M^{2}=J_{0}+J_{\beta}, \\
& M^{3}=J_{0}+J_{\alpha}, \\
& C^{2}=C^{3}=C_{0}, \\
& K^{2}=K^{3}=K_{0}, \\
& F^{2}=F^{3}=T_{e x} .
\end{aligned}
$$

Based on Equations (13)-(15), the hypothetical mode sets of substructures 2 and 3 are obtained:

$$
\varphi^{2}=\varphi^{3}=1
$$

Substructure 4 is also a flexible structure and its dynamic equation is:

$$
\begin{gathered}
{\left[\begin{array}{lll}
\mathbf{M}_{u u}^{4} & \mathbf{M}_{u C}^{4} & \mathbf{M}_{u D}^{4} \\
\mathbf{M}_{C u}^{4} & \mathbf{M}_{C C}^{4} & \mathbf{M}_{C D}^{4} \\
\mathbf{M}_{D u}^{4} & \mathbf{M}_{D C}^{4} & \mathbf{M}_{D D}^{4}
\end{array}\right]\left[\begin{array}{c}
\ddot{\mathbf{X}}_{u}^{4} \\
\ddot{\mathbf{X}}_{C}^{4} \\
\ddot{\mathbf{X}}_{D}^{4}
\end{array}\right]+\left[\begin{array}{lll}
\mathbf{C}_{u u}^{4} & \mathbf{C}_{u C}^{4} & \mathbf{C}_{u D}^{4} \\
\mathbf{C}_{C u}^{4} & \mathbf{C}_{C C}^{4} & \mathbf{C}_{C D}^{4} \\
\mathbf{C}_{D u}^{4} & \mathbf{C}_{D C}^{4} & \mathbf{C}_{D D}^{4}
\end{array}\right]\left[\begin{array}{c}
\mathbf{X}_{u}^{4} \\
\dot{\mathbf{X}}_{C}^{4} \\
\dot{\mathbf{X}}_{D}^{4}
\end{array}\right]} \\
+\left[\begin{array}{lll}
\mathbf{K}_{u u}^{4} & \mathbf{K}_{u C}^{4} & \mathbf{K}_{u D}^{4} \\
\mathbf{K}_{C u}^{4} & \mathbf{K}_{C C}^{4} & \mathbf{K}_{C D}^{4} \\
\mathbf{K}_{D u}^{4} & \mathbf{K}_{D C}^{4} & \mathbf{K}_{D D}^{4}
\end{array}\right]\left[\begin{array}{c}
\mathbf{X}_{u}^{4} \\
\mathbf{X}_{C}^{4} \\
\mathbf{X}_{D}^{4}
\end{array}\right]=\left[\begin{array}{c}
\mathbf{F}_{u}^{4} \\
\mathbf{F}_{C}^{4} \\
\mathbf{F}_{D}^{4}
\end{array}\right],
\end{gathered}
$$

in which superscript 4 represents substructure 4 in general coordinate system, subscript $u$ represents internal degree of freedom, subscript $C$ represents interface degree of freedom of interface node $C$, and subscript $D$ represents interface degree of freedom of interface node D. According to the displacement boundary conditions $\mathbf{X}_{D}^{4}=\mathbf{0}$, the dynamic equation of substructure 4 can be reduced to:

$$
\begin{gathered}
{\left[\begin{array}{cc}
\mathbf{M}_{u u}^{4} & \mathbf{M}_{u C}^{4} \\
\mathbf{M}_{C u}^{4} & \mathbf{M}_{C C}^{4}
\end{array}\right]\left[\begin{array}{l}
\ddot{\mathbf{X}}_{u}^{4} \\
\ddot{\mathbf{X}}_{C}^{4}
\end{array}\right]+\left[\begin{array}{cc}
\mathbf{C}_{u u}^{4} & \mathbf{C}_{u C}^{4} \\
\mathbf{C}_{C u}^{4} & \mathbf{C}_{C C}^{4}
\end{array}\right]\left[\begin{array}{c}
\dot{\mathbf{X}}_{u}^{4} \\
\dot{\mathbf{X}}_{C}^{4}
\end{array}\right]} \\
+\left[\begin{array}{cc}
\mathbf{K}_{u u}^{4} & \mathbf{K}_{u C}^{4} \\
\mathbf{K}_{C u}^{4} & \mathbf{K}_{C C}^{4}
\end{array}\right]\left[\begin{array}{l}
\mathbf{X}_{u}^{4} \\
\mathbf{X}_{C}^{4}
\end{array}\right]=\left[\begin{array}{c}
\mathbf{F}_{u}^{4} \\
\mathbf{F}_{C}^{4}
\end{array}\right]
\end{gathered}
$$

in which $\mathbf{M}^{4}=\left[\begin{array}{ll}\mathbf{M}_{u u}^{4} & \mathbf{M}_{u C}^{4} \\ \mathbf{M}_{C u}^{4} & \mathbf{M}_{C C}^{4}\end{array}\right], \quad \mathbf{C}^{4}=\left[\begin{array}{ll}\mathbf{C}_{u u}^{4} & \mathbf{C}_{u C}^{4} \\ \mathbf{C}_{C u}^{4} & \mathbf{C}_{C C}^{4}\end{array}\right], \quad$ and $\mathbf{K}^{4}=\left[\begin{array}{ll}\mathbf{K}_{u u}^{4} & \mathbf{K}_{u C}^{4} \\ \mathbf{K}_{C u}^{4} & \mathbf{K}_{C C}^{4}\end{array}\right]$ are mass matrix, damping matrix, and stiffness matrix of substructure 4 , respectively. When Rayleigh damping model is used, the damping matrix is:

$$
\mathbf{C}^{4}=C_{1}^{4} \mathbf{M}^{4}+C_{2}^{4} \mathbf{K}^{4}
$$

in which $C_{1}^{4}$ and $C_{2}^{4}$ are Rayleigh damping coefficients of substructure 4 and $\mathbf{X}^{4}=\left[\begin{array}{l}\mathbf{X}_{u}^{4} \\ \mathbf{X}_{C}^{4}\end{array}\right]$ and $\mathbf{F}^{4}=\left[\begin{array}{l}\mathbf{F}_{u}^{4} \\ \mathbf{F}_{C}^{4}\end{array}\right]$ are displacement column vectors and excitation column vectors, respectively. The method is given according to Equations (13)-(15). Subscript $m$ is defined as the order of main modes retained by substructure 4 , then the hypothetical mode set of substructure 4 is:

$$
\begin{aligned}
\boldsymbol{\varphi}^{4} & =\left[\begin{array}{ll}
\boldsymbol{\varphi}_{m}^{4} & \boldsymbol{\varphi}_{C}^{4}
\end{array}\right]=\left[\begin{array}{cc}
\boldsymbol{\varphi}_{u m}^{4} & \boldsymbol{\varphi}_{u C}^{4} \\
\mathbf{0}_{C m} & \mathbf{I}_{C C}
\end{array}\right], \\
\boldsymbol{\varphi}_{u C}^{4} & =-\left(\mathbf{K}_{u u}^{4}\right)^{-1} \mathbf{K}_{u C}^{4} .
\end{aligned}
$$

After obtaining the hypothetical modal sets of all substructures, the mass matrix, damping matrix, stiffness matrix, 
displacement column vector, excitation column vector, and mode matrix of the system can be defined as:

$$
\begin{aligned}
& \mathbf{M}=\left[\begin{array}{llll}
\mathbf{M}^{b} & & & \\
& & & \\
& M^{2} & & \\
& & M^{3} & \\
& & & \mathbf{M}^{4}
\end{array}\right], \\
& \mathbf{C}=\left[\begin{array}{llll}
\mathbf{C}^{b} & & & \\
& & & \\
& C^{2} & & \\
& & C^{3} & \\
& & & \\
& & & \mathbf{C}^{4}
\end{array}\right] \text {, } \\
& \mathbf{M}_{p} \ddot{\mathbf{X}}_{p}+\mathbf{C}_{p} \dot{\mathbf{X}}_{p}+\mathbf{K}_{p} \mathbf{X}_{p}=\mathbf{F}_{p} \text {, } \\
& \mathbf{M}_{p}=\boldsymbol{\varphi}^{T} \mathbf{M} \boldsymbol{\varphi}, \\
& \mathrm{C}_{p}=\varphi^{T} \mathbf{C} \boldsymbol{\varphi} \\
& \mathbf{K}_{p}=\boldsymbol{\varphi}^{T} \mathbf{K} \boldsymbol{\varphi}, \\
& \mathbf{F}_{p}=\varphi^{T} \mathbf{F} \text {. }
\end{aligned}
$$$$
\mathbf{K}=\left[\begin{array}{llll}
\mathbf{K}^{b} & & & \\
& K^{2} & & \\
& & K^{3} & \\
& & & \mathbf{K}^{4}
\end{array}\right]
$$$$
\mathbf{X}=\left[\begin{array}{c}
\mathbf{X}^{b} \\
\beta \\
\alpha \\
\mathbf{X}^{4}
\end{array}\right],
$$$$
\mathbf{F}=\left[\begin{array}{c}
\mathbf{F}^{b} \\
\mathbf{F}^{2} \\
\mathbf{F}^{3} \\
\mathbf{X}^{4}
\end{array}\right],
$$$$
\varphi=\left[\begin{array}{llll}
\varphi^{b} & & & \\
& \varphi^{2} & & \\
& & \varphi^{3} & \\
& & & \\
& & & \varphi^{4}
\end{array}\right] .
$$

The modal coordinate $\mathbf{X}_{p}$ corresponding to the modal matrix $\varphi$ can be written as:

$$
\mathbf{X}_{p}=\left[\begin{array}{llllll}
\mathbf{p}_{l}^{b} & \mathbf{X}_{A}^{b} & \beta & \alpha & \mathbf{p}_{m}^{4} & \mathbf{X}_{C}^{4}
\end{array}\right]^{T},
$$

in which $\mathbf{p}_{l}^{b}$ and $\mathbf{p}_{m}^{4}$ are the modal coordinates of substructures 1 and 4, and the relationship between the modal coordinates $\mathbf{X}$ and the physical coordinates $\mathbf{X}_{p}$ is:

$$
\mathbf{X}=\varphi \mathrm{X}_{p}
$$

By the first coordinate transformation of the dynamic equation of the system, the physical coordinates with high degree of freedom of the coupled system can be reduced into mixed coordinates with low degree of freedom, i.e., the

Substituting coordinate transformation relation given by Equation (12) and interface displacement coordination condition:

$$
\begin{aligned}
\mathbf{X}_{A}^{b} & =\mathbf{R}_{\beta}{ }^{T} \mathbf{R}_{\alpha}{ }^{T} \mathbf{X}_{A}, \\
\mathbf{X}_{C}^{4} & =\mathbf{X}_{C}, \\
\mathbf{X}_{A} & =\mathbf{X}_{C}+\mathbf{I}_{4} \alpha+\mathbf{I}_{5} \beta, \\
\mathbf{I}_{4} & =\left(\begin{array}{llllll}
0 & 0 & 0 & 1 & 0 & 0
\end{array}\right)^{T}, \\
\mathbf{I}_{5} & =\left(\begin{array}{llllll}
0 & 0 & 0 & 0 & 1 & 0
\end{array}\right)^{T} .
\end{aligned}
$$

However, the modal coordinates $\mathbf{X}_{p}$ are not completely independent. By substituting transformation matrix $\mathbf{S}$, the nonindependent coordinates in $\mathbf{X}_{p}$ are eliminated and the independent mode coordinates $\mathbf{X}_{q}$ of the coupling system are obtained:

$$
\begin{gathered}
\mathbf{X}_{p}=\mathbf{S X}_{q}, \\
\mathbf{X}_{q}=\left[\begin{array}{c}
\mathbf{p}_{l}^{b} \\
\mathbf{p}_{m}^{4} \\
\mathbf{X}_{C} \\
\alpha \\
\beta
\end{array}\right],
\end{gathered}
$$

$$
\mathbf{S}=\left[\begin{array}{ccccc}
\mathbf{I} & \mathbf{0} & \mathbf{0} & \mathbf{0} & \mathbf{0} \\
\mathbf{0} & \mathbf{0} & \mathbf{R}_{\beta}{ }^{T} \mathbf{R}_{\alpha}{ }^{T} & \mathbf{R}_{\beta}{ }^{T} \mathbf{R}_{\alpha}{ }^{T} \mathbf{I}_{4} & \mathbf{R}_{\beta}{ }^{T} \mathbf{R}_{\alpha}{ }^{T} \mathbf{I}_{5} \\
\mathbf{0} & \mathbf{0} & \mathbf{0} & 0 & 1 \\
\mathbf{0} & \mathbf{0} & \mathbf{0} & 1 & 0 \\
\mathbf{0} & \mathbf{I} & \mathbf{0} & \mathbf{0} & \mathbf{0} \\
\mathbf{0} & \mathbf{0} & \mathbf{I} & \mathbf{0} & \mathbf{0}
\end{array}\right]
$$


TABLE 1: Modal frequency and mode shape of the data transmission antenna.

\begin{tabular}{lcc}
\hline Order & Frequency/Hz & Mode shapes \\
\hline 1 & 7.924 & The combination torsion around $Z$-axis \\
2 & 12.209 & First-order bending of the support arm in $X Z$ plane \\
3 & 17.860 & First-order bending of the support arm in $Y Z$ plane \\
4 & 52.337 & Tension of the combination along the $Z$-axis \\
5 & 89.975 & Second-order bending of the support arm in $X Z$ plane \\
6 & 112.781 & Second-order bending of the support arm in $Y Z$ plane \\
7 & 118.847 & Bending of the reflector \\
\hline
\end{tabular}

Then, the dynamic equation of the coupled system expressed in independent mode coordinates with lower degrees of freedom can be written as follows:

$$
\mathbf{M}_{q} \ddot{\mathbf{X}}_{q}+\mathbf{C}_{q} \dot{\mathbf{X}}_{q}+\mathbf{K}_{q} \mathbf{X}_{q}=\mathbf{F}_{q}
$$

in which

$$
\begin{aligned}
\mathbf{M}_{q} & =\mathbf{S}^{T} \mathbf{M}_{p} \mathbf{S}, \\
\mathbf{C}_{q} & =\mathbf{S}^{T} \mathbf{C}_{p} \mathbf{S}, \\
\mathbf{K}_{q} & =\mathbf{S}^{T} \mathbf{K}_{p} \mathbf{S}, \\
\mathbf{F}_{q} & =\mathbf{S}^{T} \mathbf{F}_{p} .
\end{aligned}
$$

Equation (31) has $(l+m+8)$-order degrees of freedom; coordinates are independent of each other; and $\mathbf{X}_{C}, \alpha$, and $\beta$ have clear physical meanings. It is convenient to establish the electromechanical coupling model of the data transmission antenna by combining the control model. At the same time, the lower degree of freedom guarantees the efficiency of iteration calculation and the independent coordinates are convenient to analyze the coupling relationship. In order to facilitate the connection between the dynamic model and the control model, the subscript $s$ is used to represent the coupling structure, and Equation (31) is rewritten into the state space form:

$$
\left\{\begin{array}{l}
\dot{\mathbf{X}}_{s}=\mathbf{A}_{s} \mathbf{X}_{s}+\mathbf{B}_{s} \mathbf{U}_{s}, \\
\mathbf{Y}_{s}=\mathbf{C}_{s} \mathbf{X}_{s},
\end{array}\right.
$$

in which

$$
\begin{aligned}
& \mathbf{X}_{s}=\left[\begin{array}{c}
\mathbf{X}_{q} \\
\dot{\mathbf{X}}_{q}
\end{array}\right], \\
& \mathbf{A}_{s}=\left[\begin{array}{cc}
\mathbf{0} & \mathbf{I} \\
-\mathbf{M}_{q}^{-1} \mathbf{C}_{q} & -\mathbf{M}_{q}^{-1} \mathbf{K}_{q}
\end{array}\right], \\
& \mathbf{B}_{s}=\left[\begin{array}{c}
\mathbf{0} \\
\mathbf{M}_{q}^{-1}
\end{array}\right],
\end{aligned}
$$

$$
\begin{aligned}
& \mathbf{U}_{s}=\mathbf{F}_{q}, \\
& \mathbf{Y}_{s}=\left[\begin{array}{l}
\alpha \\
\beta
\end{array}\right], \\
& \mathbf{C}_{s}=\left[\begin{array}{l}
0 \\
1 \\
1
\end{array}\right] .
\end{aligned}
$$

The state variable $\mathbf{X}_{s}$ is composed of the independent modal coordinates $\mathbf{X}_{q}$ and their derivatives. The system matrix $\mathbf{A}_{s}$ is composed of transformed mass matrix, damping matrix, and stiffness matrix, which reflects the dynamic characteristics of the coupling structure. The input vector $\mathbf{U}_{s}$ consists of the input force of two stepping motors, and the input transformation matrix $\mathbf{B}_{s}$ reflects the position of the input force and mode shape information. The output variable $\mathbf{Y}_{s}$ is composed of the angular displacement of the stepping motors. As the feedback of the closed-loop control of the angular displacement of the motors, the output transformation matrix $\mathbf{C}_{s}$ can extract the required output variables from the state variables. The order of the state space equation of the data transmission antenna is low, which improves the simulation efficiency. Moreover, the state space equation can be easily connected with the control model of the twoaxis motors for cosimulation to optimize the control method and control parameters.

2.3. Microvibration Model and Coupling Relationship Analysis of the Antenna. When the stepper motor of the two-axle antenna is started, the driving system will input pulse current to the motor, which will cause vibration of the planetary gear during meshing. The fundamental frequency $f_{0}$ of vibration caused by current pulses is:

$$
f_{0}=\frac{\omega h}{\gamma_{z}}
$$

in which $\omega$ is the speed of the stepping motor, $h$ is the deceleration ratio, and $\gamma_{z}$ is the mechanical step angle of the stepping motor. According to Equation (6), the mechanical step angle is related to the running beats, the subdivisions number, and the number of rotor teeth of the stepping motor. In addition, the planetary gear will generate multiple 


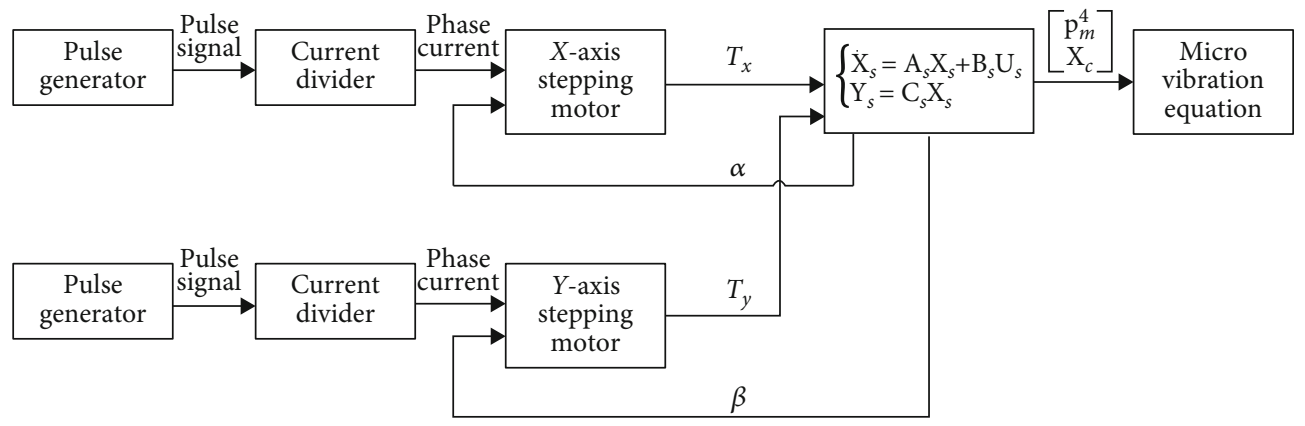

Figure 3: Simulation model of data transmission antenna.

TABLE 2: Simulation parameters.

\begin{tabular}{|c|c|c|c|}
\hline Parameter symbol & Physical meaning & Value & Unit \\
\hline$R$ & Phase resistance & 6.5 & $\Omega$ \\
\hline$L_{0}$ & Phase inductance & 30 & $\mathrm{mH}$ \\
\hline$I_{m}$ & Amplitude of two-phase current & 0.6 & A \\
\hline$n$ & The subdivisions number & 8 & - \\
\hline$\gamma_{z}$ & Mechanical step angle & $\pi / 100$ & rad \\
\hline$z$ & Rotor teeth & 50 & - \\
\hline$J_{0}$ & Motor rotor inertia & 0.0001 & $\mathrm{~kg} \cdot \mathrm{m}^{2}$ \\
\hline$C_{0}$ & Viscous damping factor of the motor & 0.02 & $\mathrm{~N} \cdot \mathrm{m} \cdot \mathrm{s} / \mathrm{rad}$ \\
\hline$K_{m}$ & Electromagnetic moment coefficient & 0.16 & $\mathrm{~N} \cdot \mathrm{m} / \mathrm{A} \cdot \mathrm{rad}$ \\
\hline$h$ & Deceleration ratio & 100 & - \\
\hline- & Total quality of the antenna & 25 & $\mathrm{~kg}$ \\
\hline- & Rotating inertia driven by $x$-axis motor & 0.34 & $\mathrm{~kg} \cdot \mathrm{m}^{2}$ \\
\hline- & Rotating inertia driven by $y$-axis motor & 0.0925 & $\mathrm{~kg} \cdot \mathrm{m}^{2}$ \\
\hline$C_{1}^{b} C_{1}^{4}$ & Rayleigh damping coefficient & 0.001 & - \\
\hline$C_{2}^{b} C_{2}^{4}$ & Rayleigh damping coefficient & 0.0005 & - \\
\hline
\end{tabular}

frequency doubling excitation along with the fundamental frequency.

The microvibration generated by the coupling system of the dual-axis data transmission antenna can be calculated by the reaction force generated by the coupling system on the fixed interface, i.e., the supporting reaction force at node $\mathrm{D}$. The microvibration generated by the coupling system of two-axis digital transmission antenna can be calculated by the reaction force generated by the coupling system on the fixed interface, that is, the supporting reaction force at node $\mathrm{D}$. In the third line of Equation (20) and substituting the displacement boundary conditions $\mathbf{X}_{D}^{4}=0$, the calculation formula of microvibration of coupled system can be obtained:

$$
\begin{aligned}
\mathbf{F}_{m}= & \mathbf{F}_{D}^{4}=\left[\begin{array}{ll}
\mathbf{M}_{D u}^{4} & \mathbf{M}_{D C}^{4}
\end{array}\right] \ddot{\mathbf{X}}^{4}+\left[\begin{array}{ll}
\mathbf{C}_{D u}^{4} & \mathbf{C}_{D C}^{4}
\end{array}\right] \dot{\mathbf{X}}^{4} \\
& +\left[\begin{array}{ll}
\mathbf{K}_{D u}^{4} & \mathbf{K}_{D C}^{4}
\end{array}\right] \mathbf{X}^{4},
\end{aligned}
$$

$$
\mathbf{X}^{4}=\left[\begin{array}{l}
\mathbf{X}_{u}^{4} \\
\mathbf{X}_{C}^{4}
\end{array}\right]=\varphi^{4}\left[\begin{array}{l}
\mathbf{p}_{m}^{4} \\
\mathbf{X}_{C}
\end{array}\right]
$$

According to Equation (27), the specific expressions of mass matrix and stiffness matrix in the dynamic equation after polycondensation are as follows:

$$
\mathbf{M}_{p}=\left[\begin{array}{cccccc}
\mathbf{I}_{l l} & \mathbf{M}_{l A}^{b} & & & & \\
\mathbf{M}_{A l}^{b} & \mathbf{M}_{A A}^{b} & & & & \\
& & J_{0}+J_{\beta} & & & \\
& & & J_{0}+J_{\alpha} & & \\
& & & \mathbf{I}_{m m} & \mathbf{M}_{m C}^{4} \\
& & & \mathbf{M}_{C m}^{4} & \mathbf{M}_{C C}^{4}
\end{array}\right],
$$




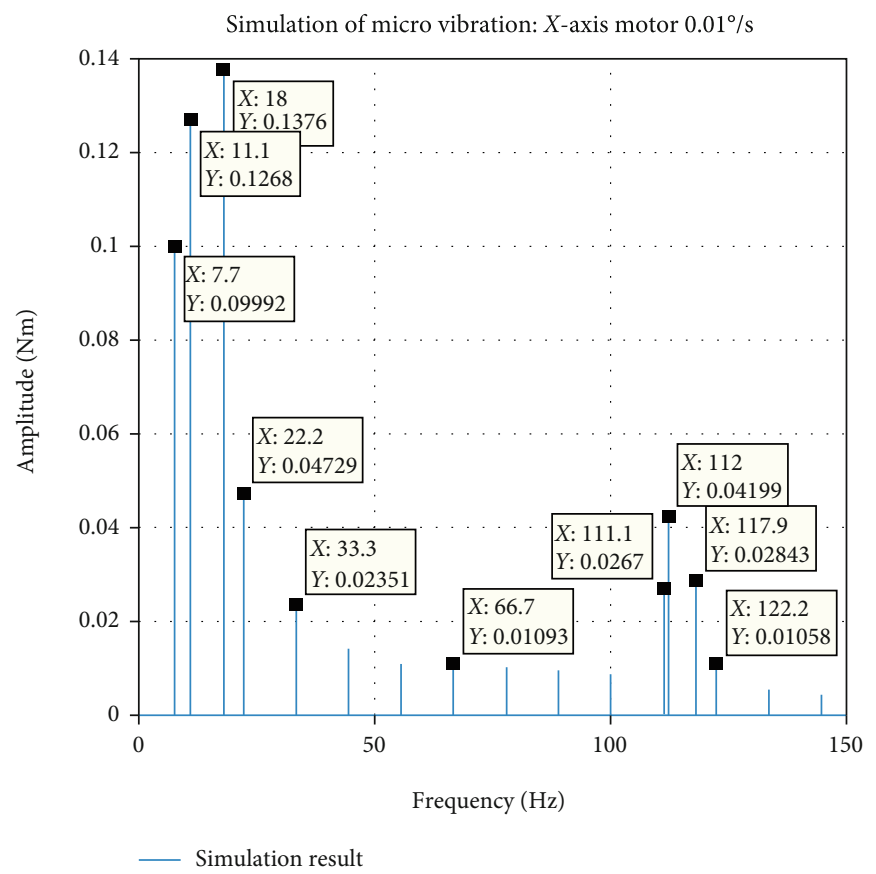

(a)

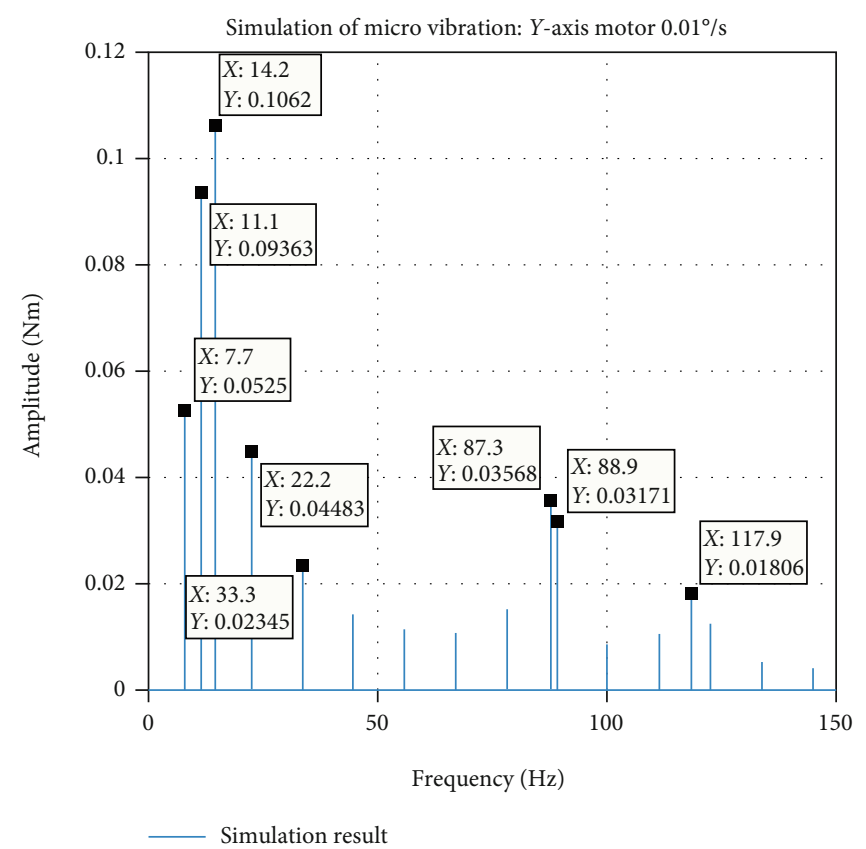

(b)

FIGURE 4: Simulation results of microvibration frequency domain curve: (a) $x$-axis motor; (b) $y$-axis motor.

$$
\mathbf{K}_{p}=\left[\begin{array}{cccccc}
\boldsymbol{\Lambda}_{l l} & & & & & \\
& \mathbf{K}_{A A}^{b} & & & & \\
& & J_{0}+J_{\beta} & & & \\
& & & J_{0}+J_{\alpha} & & \\
& & & & \boldsymbol{\Lambda}_{m m} & \\
& & & & & \mathbf{K}_{C C}^{4}
\end{array}\right]
$$

The stiffness matrix $\mathbf{K}_{p}$ is diagonal matrix, which indicates that the dynamic equation of the system after polycondensation is decoupled on stiffness. $\boldsymbol{\Lambda}_{l l}=\operatorname{diag}\left(2 \pi f_{i}\right), i=1,2, \cdots, l$ and $\boldsymbol{\Lambda}_{m m}=\operatorname{diag}\left(2 \pi f_{j}\right), j=1,2, \cdots, m$ are diagonal matrices, representing the retained main modes frequency of the substructure 1 reflector and the substructure 4 support arm, respectively. The mass matrix $\mathbf{M}_{p}$ is a symmetrical nondiagonal matrix, which indicates that the dynamic equation of the system is coupled on mass. $\mathbf{M}_{l A}^{b}=\left(\mathbf{M}_{A l}^{b}\right)^{T}$ reflects the coupling 


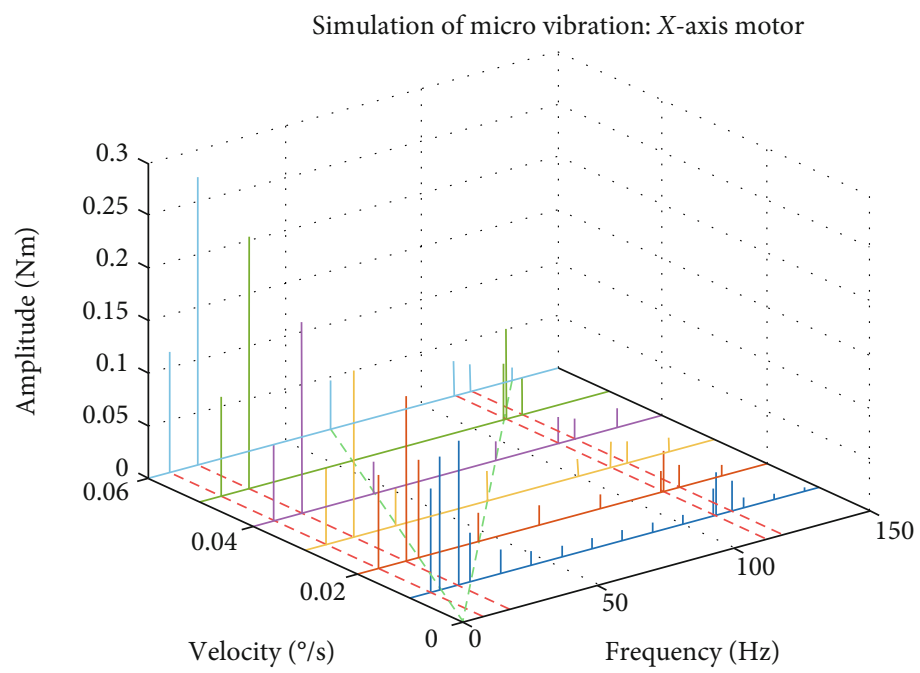

(a)

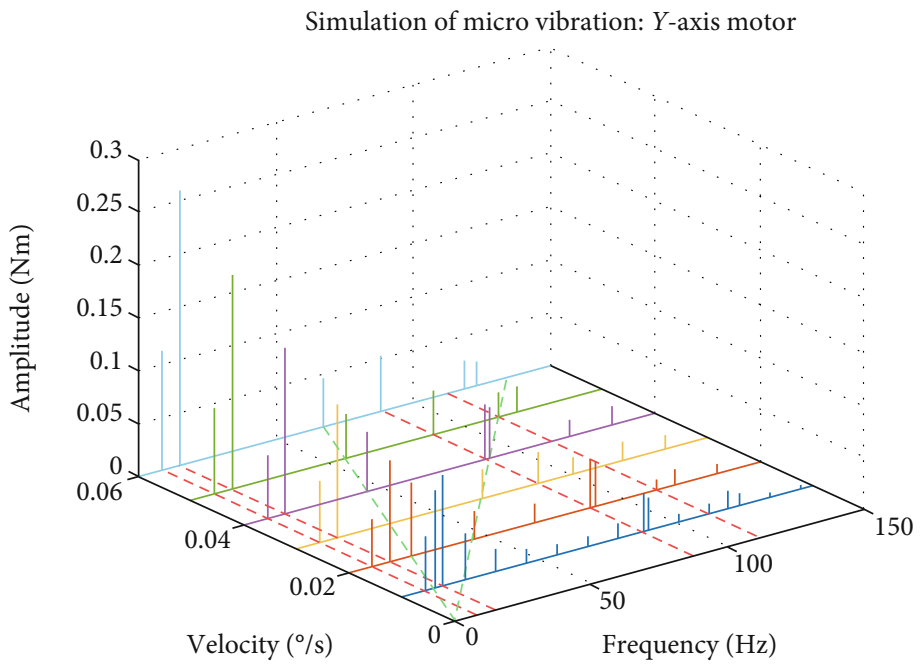

(b)

FIGURE 5: Simulation results of microvibration of the antenna at different velocity: (a) $x$-axis motor; (b) $y$-axis motor.

relationship between the modes of the flexible load and the physical coordinates of the system, and $\mathbf{M}_{m C}^{4}=\left(\mathbf{M}_{C m}^{4}\right)^{T}$ reflects the coupling relationship between the modes of the flexible boundary and the physical coordinates of the system.

\section{Simulation Analysis of the System Using MATLAB/Simulink Toolbox}

3.1. Simulation Model of the Dual-Axis Data Transmission Antenna. The dynamic behavior of the coupling structure of the data transmission antenna can be preliminarily analyzed through the simulation. Firstly, the mode of the data transmission antenna is obtained by modal experiment. The modal frequency and mode shape of the data transmission antenna are given in Table 1 . According to the modal experiment results, the finite element model of the data transmission antenna is established. Then, the mass matrix and the stiffness matrix of the flexible structure reflector and support arm are extracted from the finite element model.
The state space form of coupled system dynamics equation is obtained according to the method given in the second section. The simulation model of digital antenna is established by using the Simulink toolbox of commercial software MATLAB (as shown in Figure 3). Table 2 gives the parameters used in the simulation process.

3.2. Simulation Results and Analysis. The simulation program can output the time domain and frequency domain results of the microvibration caused by the coupled structure, but the frequency domain results of the microvibration are more concerned in practice. The simulation results of the microvibration caused by the $x$-axis motor and $y$-axis motor of the coupled structure are given in Figures 4 and 5. When the $x$-axis motor rotates, natural frequencies of $7.7 \mathrm{~Hz}$, $18 \mathrm{~Hz}, 112 \mathrm{~Hz}$, and $117.9 \mathrm{~Hz}$ appear at different velocity conditions, corresponding to $7.9 \mathrm{~Hz}, 17.8 \mathrm{~Hz}, 112.7 \mathrm{~Hz}$, and $118.8 \mathrm{~Hz}$ of the first, third, sixth, and seventh order mode frequencies of the structure, respectively, and the mode shapes of these modes all have components in the direction 


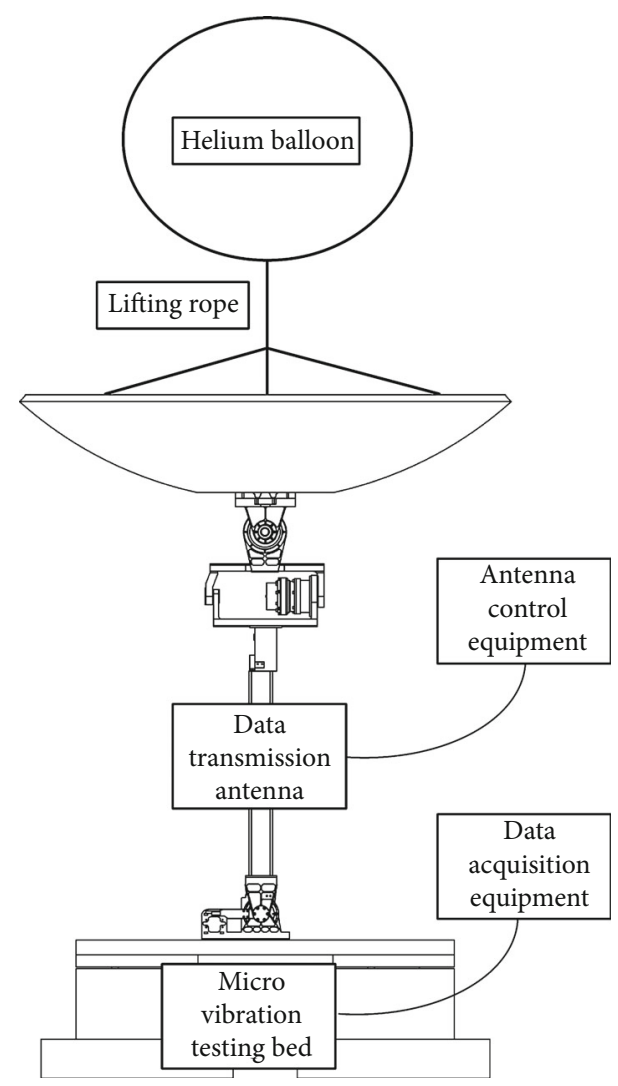

Figure 6: Microvibration experiment of the data transmission antenna.

of $x$-axis motor rotation. On the other hand, when the $y$-axis motor rotates, natural frequencies of $7.7 \mathrm{~Hz}, 14 \mathrm{~Hz}, 87.3 \mathrm{~Hz}$, and $117.9 \mathrm{~Hz}$ appear at different velocity conditions, corresponding to $7.9 \mathrm{~Hz}, 12.2 \mathrm{~Hz}, 89.9 \mathrm{~Hz}$, and $118.8 \mathrm{~Hz}$ of the first, second, fifth, and seventh order modal frequencies of the structure, respectively, and the mode shapes of these modes all have components in the direction of $y$-axis motor rotation.

At the same time, the simulation results of microvibration of two motors at different velocity not only show the fundamental frequency caused by the current pulse satisfying Equation (35), such as $11.11 \mathrm{~Hz}, 22.22 \mathrm{~Hz}, 33.33 \mathrm{~Hz}$, $44.44 \mathrm{~Hz}, 55.56 \mathrm{~Hz}$, and $66.67 \mathrm{~Hz}$, but also multiple frequency doubling accompanied by the fundamental frequency. According to the amplitude of each frequency, the amplitude of microvibration caused by fundamental frequency of current pulse is larger, while the amplitude of microvibration caused by frequency doubling shows a tendency to decrease with the increase of frequency as a whole. However, if the frequency doubling approaches the structural mode frequency, the coupling phenomenon of common amplification will occur.

\section{Experimental Tests of the Antenna in Simulated Zero Gravity State}

4.1. Experimental System. The experimental system consists of three parts: equipment to be tested, test equipment, and
TABLE 3: Suspension frequency of zero gravity simulator.

\begin{tabular}{lcccc}
\hline Object & $T / \mathrm{N}$ & $m / \mathrm{kg}$ & $L / \mathrm{m}$ & $f_{\mathrm{He}} / \mathrm{Hz}$ \\
\hline$x$-axis motor & 79.56 & 0.53 & 2.96 & 0.658 \\
$y$-axis motor & 60.20 & 0.53 & 2.96 & 0.573 \\
\hline
\end{tabular}

zero gravity state simulation equipment (as shown in Figure 6). The equipment to be tested are the data transmission antenna and the antenna control equipment. The test equipment includes the microvibration testing bed, unidirectional force sensor, acceleration sensor, and force hammer. The microvibration testing bed is a high rigidity piezoelectric force testing bed developed by Beihang University. Its testing principle and method are given in manuscript [25]. It is used to measure the microvibration of the coupling system. When the helium balloon is inflated, the unidirectional force sensor measures the tension of the lifting rope of the helium balloon. The acceleration sensor and the force hammer are used to test the antenna mode.

In order to simulate the zero gravity state of the satelliteborne data transmission antenna in orbit, the helium balloon is used to balance the load gravity of the motor shaft, and the zero gravity state of the motor shaft is simulated. The simulation equipment also includes lifting rope, lifting ring installed in antenna reflector and protective rope. During the experiment, the helium balloon was suspended from the antenna reflector by the lifting rope. The laboratory is a confined space, and the air conditioner and other equipment that can cause air flow are closed during the experiment, so the influence of air flow on the experiment can be ignored. The suspended helium balloon can be equivalent to a pendulum model, and its suspension frequency $f_{\mathrm{He}}$ is as follows:

$$
f_{\mathrm{He}}=\frac{1}{2 \pi} \sqrt{\frac{T}{m L^{2}}},
$$

in which $T$ is the rope tension, $m$ is the mass of the helium balloon when it is not inflated, and $L$ is the distance from the lifting point to the balloon centroid. The balloon mass and the length of the lifting rope are measured before the experiment, and the tension of the lifting rope is measured by a unidirectional force sensor during the experiment. The suspension frequency to simulate the zero gravity state of the $x$-axis and $y$-axis motors is calculated (as shown in Table 3 ). The suspension frequency is much lower than the first mode frequency of the coupling structure, so the influence of the helium balloon shaking on the experiment can be ignored.

4.2. Experimental Results and Coupling Analysis. Figure 7 shows the comparison of experimental results and simulation results of microvibration frequency domain curve. Tables 4 and 5 show the comparison of experimental results and simulation results of microvibration amplitude. Through comparison, the frequency of microvibration obtained by experiment and simulation is consistent, and the amplitude error of main frequency is less than $6 \%$. Therefore, the 


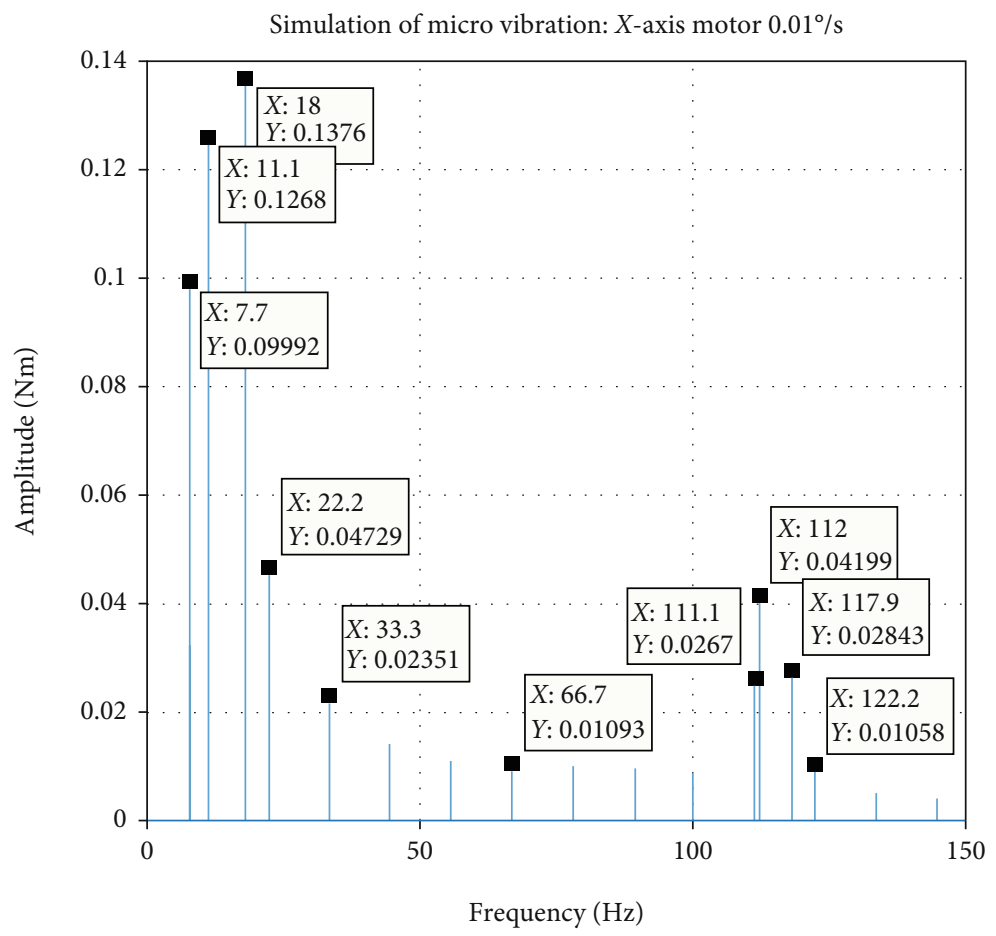

— Simulation result

(a)

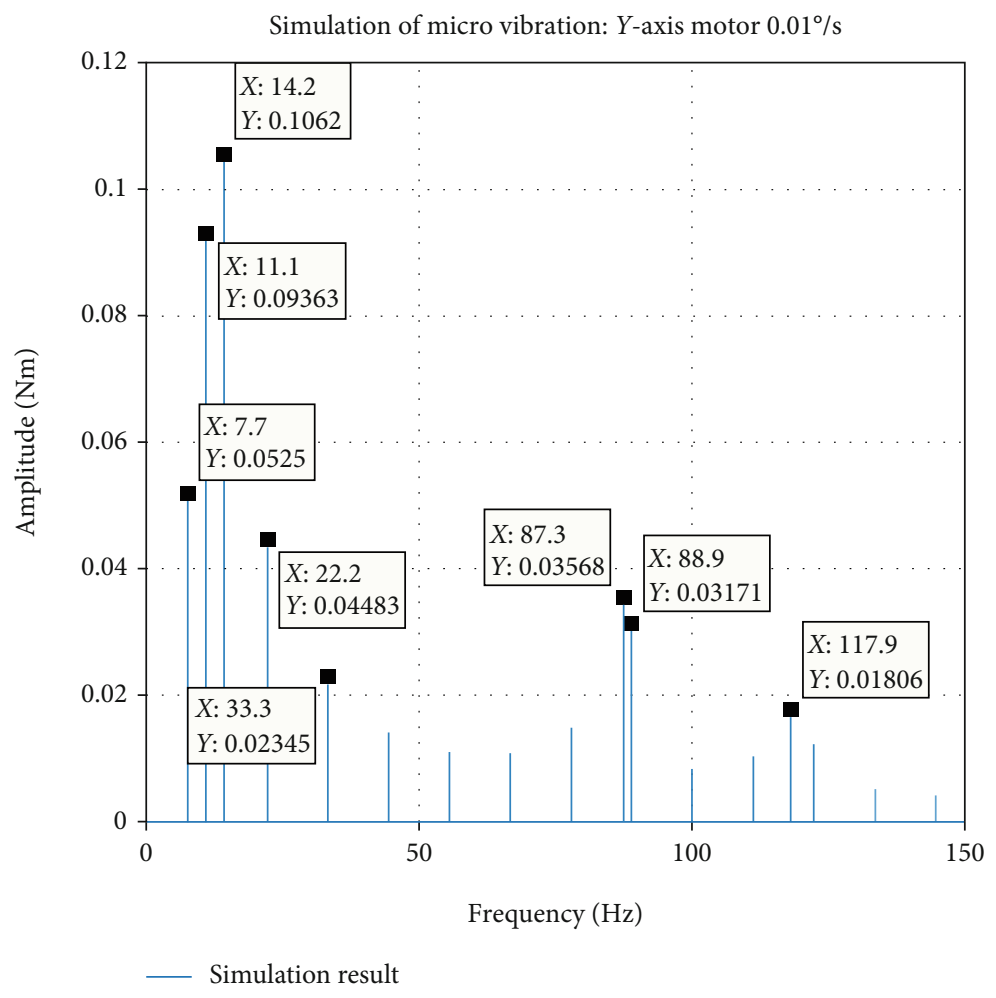

(b)

FIgURE 7: Continued. 


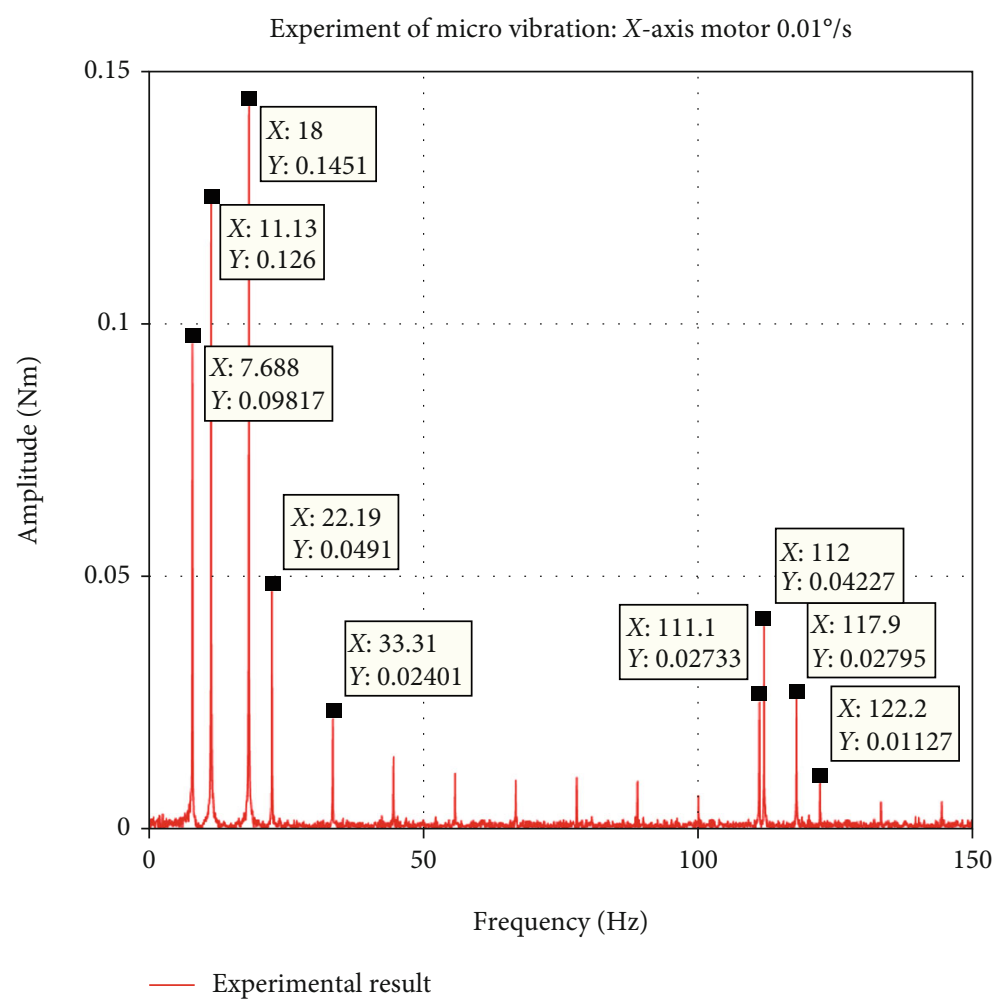

(c)

Experiment of micro vibration: $Y$-axis motor $0.01 \%$

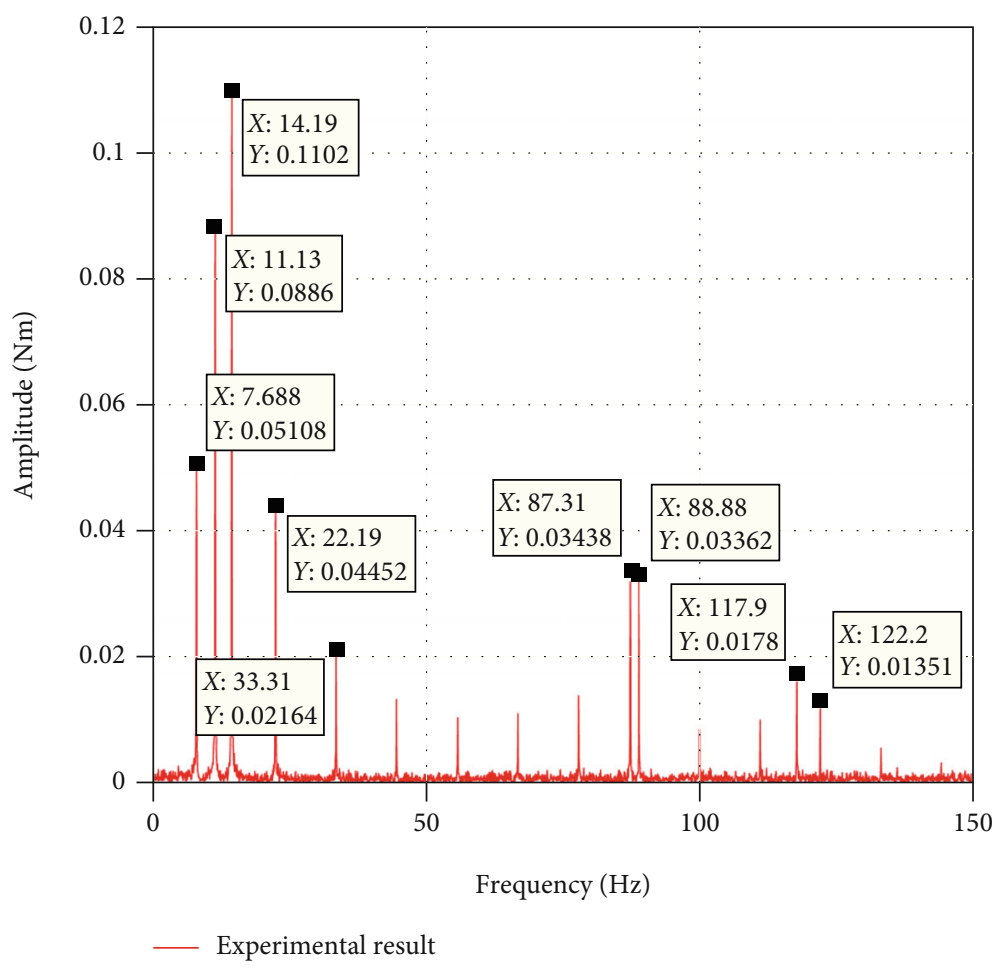

(d)

FiguRE 7: Simulation results of microvibration frequency domain curve: (a) $x$-axis motor, (b) $y$-axis motor and experimental results of microvibration frequency domain curve: (c) $x$-axis motor, (d) $y$-axis motor. 


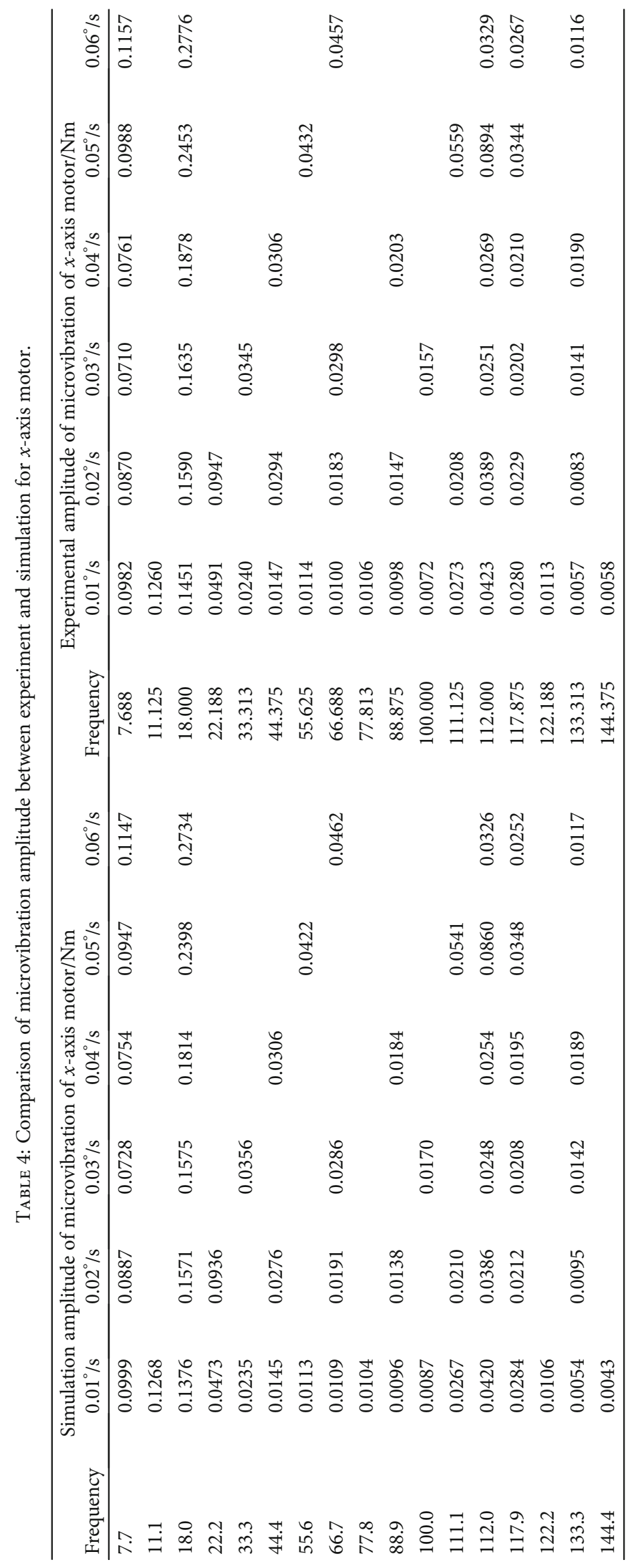




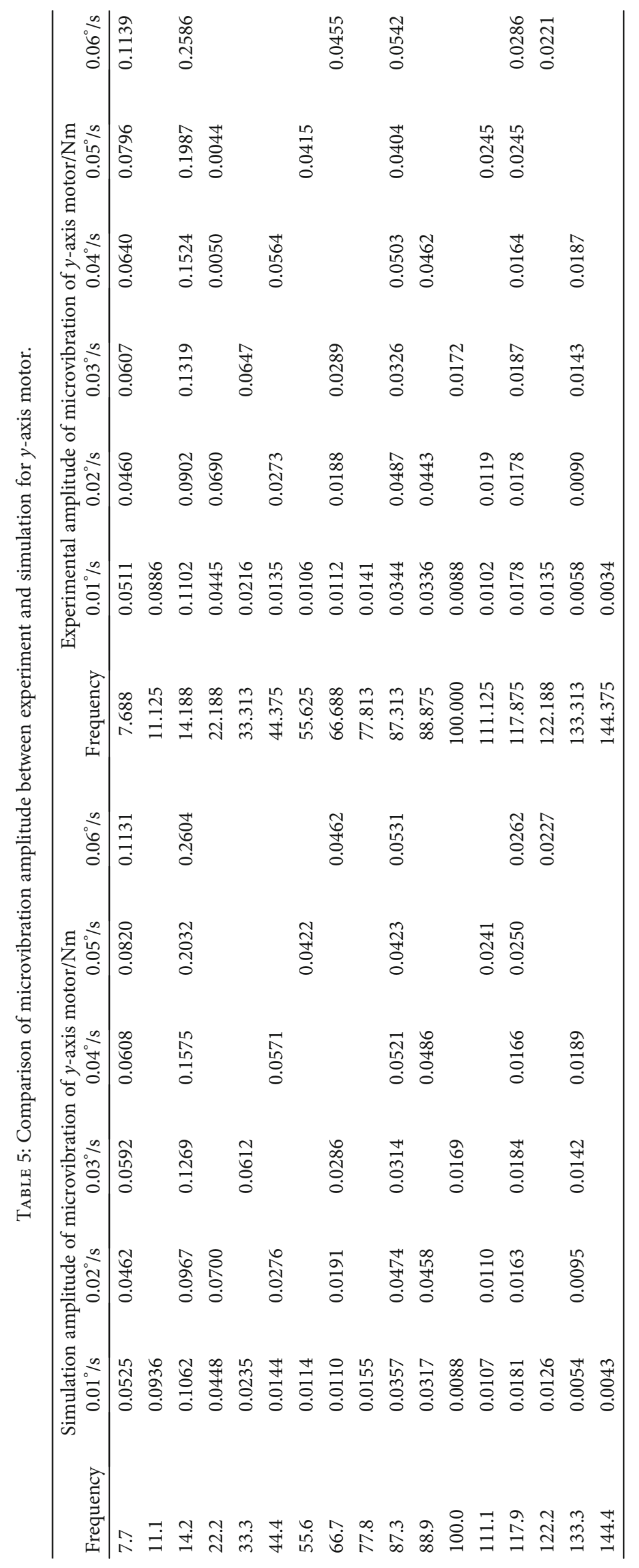




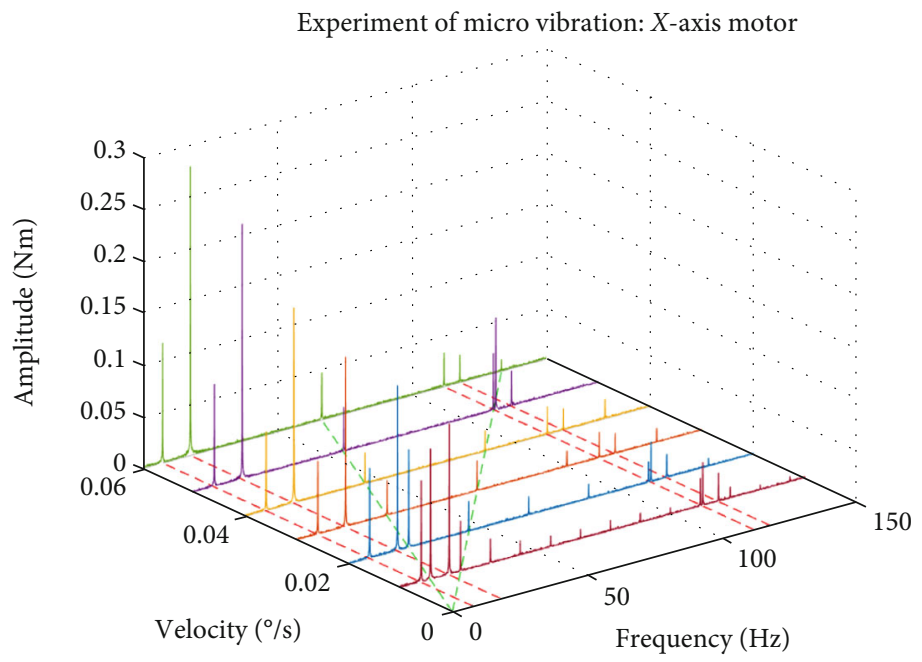

(a)

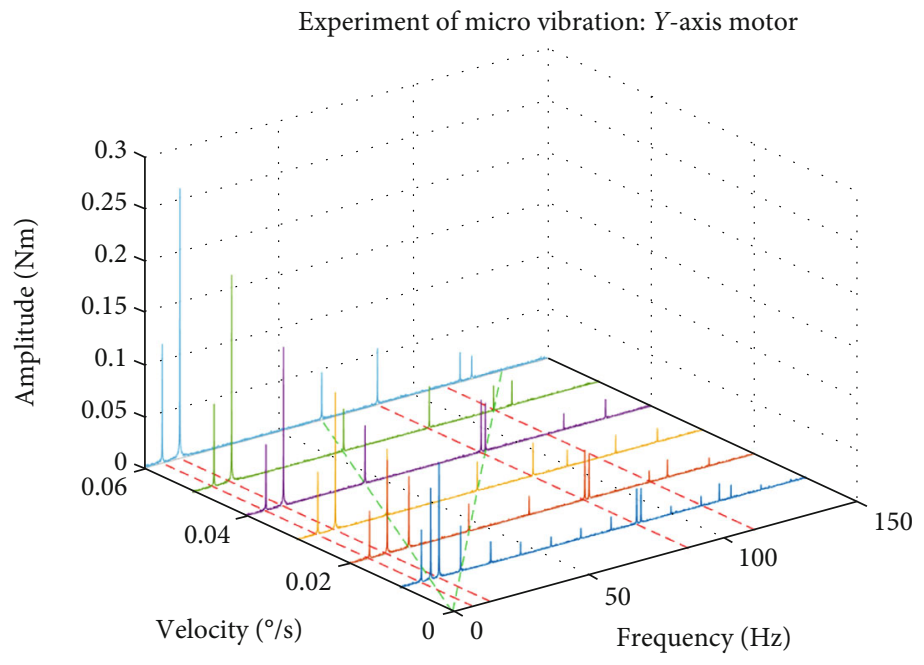

(b)

FIGURE 8: Experimental results of microvibration of the antenna at different velocity: (a) $x$-axis motor; (b) $y$-axis motor.

microvibration model given in Section 2 can accurately predict the microvibration of the coupled system.

Taking the experimental results as reference, the simulation results have deviation. The possible reasons are the deviation caused by simplification and linearization of electromagnetic torque, the vibration and fluctuation of real current, the error caused by the instability of motor system, the test error of the microvibration testing bed, and the random interference of the experimental process.

According to the frequency domain curve of microvibration, the main causes of microvibration are as follows:

(1) The rotation of the stepping motor causes the coupling structure to generate microvibration at the modal frequency with the rotational direction component

(2) Electromagnetic excitation generated by the pulse exciting current
(3) In the meshing process of planetary gear, the frequency doubling excitation of pulse excitation is produced

Figure 8 shows the experimental results of microvibration of the antenna at different velocity. Combined with the simulation results, it can be seen that there is electromechanical coupling between the stepping motor and the flexible load or flexible boundary. The specific performance is that the electromagnetic stiffness of the stepping motor will affect the microvibration frequency of the coupling structure, which deviates from the original structure frequency.

When the velocity of stepper motor is low, the coupling structure has more resonance frequency in low-frequency band. If the fundamental frequency and frequency doubling of the microvibration caused by the pulse exciting current are close to the natural frequency of the coupling structure, the coupling phenomenon of amplifying the amplitude of the microvibration will occur. As the velocity of the stepper 
motor increases, the fundamental frequency and frequency multiplication of the microvibration caused by the pulsed excitation current increase, the resonance frequency in the low frequency band decreases, and the probability of coupling with the structure frequency decreases. However, when the velocity is high, the low-frequency microvibration amplitude of the coupling structure increases significantly.

Therefore, in order to reduce the microvibration caused by the coupling structure, the appropriate velocity should be selected as the working velocity. The working velocity should not only make the frequency of microvibration caused by pulse current avoid the structure frequency which may cause coupling but also avoid too fast velocity to cause large low-frequency vibration.

\section{Conclusions}

In this study, the microvibration phenomena of the stepper motors driving the flexible load on the flexible boundary are analyzed. A dynamic equation and a microvibration model of the dual-axis data transmission antenna oriented to the control system are given by simplifying the stepper motor electromagnetic moment and the Dynamic Substructure Method. The coupling relationship between the stepping motors and the flexible structure is analyzed through the transformation of modal coordinates. The simulation and experimental results show that the model can accurately predict the microvibration of the coupling structure, and the main causes of the microvibration are the electromechanical coupling of the stepper motors and the flexible structure, electromagnetic pulse excitation, and planetary gear meshing. The influence of velocity of the motors on the output microvibration of the coupling structure is studied. It is pointed out that the influence of the working velocity, excitation torque, load torque, mechanical step angle, and other parameters should be carefully considered in the antenna design to avoid coupling between microvibration and structure frequency.

This study can be used to predict the microvibration of remote sensing satellite in orbit and provide a research basis for the design of the antenna control system and the vibration suppression of remote sensing satellite in orbit.

\section{Data Availability}

The available data are given in the manuscript.

\section{Conflicts of Interest}

The authors declare that they have no conflicts of interest.

\section{Acknowledgments}

The research is funded by the China High Resolution Earth Observation Project.

\section{References}

[1] M. Xia, C. Qin, X. Wang, and Z. Xu, "Modeling and experimental study of dynamic characteristics of the moment wheel assembly based on structural coupling," Mechanical Systems and Signal Processing, vol. 146, article 107007, 2021.

[2] S. Chen, M. Xuan, J. Xin et al., "Design and experiment of dual micro-vibration isolation system for optical satellite flywheel," International Journal of Mechanical Sciences, vol. 179, article 105592, 2020.

[3] X. Li and W. Cheng, "Research on microvibrations generated by a control moment gyroscope on a flexible interface based on a dynamic substructure method," International Journal of Aerospace Engineering, vol. 2018, Article ID 5045740, 17 pages, 2018.

[4] Z. Zheng, W. Cheng, and G. Wang, "Analysis and experimental study on dynamic behavior of permanent magnet synchronous motor in driving flexible solar array," Journal of Vibration and Control, vol. 27, no. 7-8, pp. 943-956, 2021.

[5] X. Wang, Z. Xu, S. He et al., "Modeling and analysis of a multidegree-of-freedom micro-vibration simulator," Shock and Vibration, vol. 2017, Article ID 4840514, 17 pages, 2017.

[6] J. Tao, T. Zhang, and Y. Nie, "Attitude maneuvering and vibration reducing control of flexible spacecraft subject to actuator saturation and misalignment," Shock and Vibration, vol. 2018, Article ID 3129834, 16 pages, 2018.

[7] X. Wei, L. He, and L. Chen, "Retrodirective antenna for intersatellite data transmission," IEEE Access, vol. 8, pp. 8972089726, 2020.

[8] S. D. Silverstein, J. M. Ashe, G. M. Kautz, F. W. Wheeler, and A. Jacomb-Hood, "Tripulse: a system for determining orientation and attitude of a satellite borne active phased array," IEEE Transactions on Aerospace and Electronic Systems, vol. 38, no. 1, pp. 2-12, 2002.

[9] L. Li, R. Shi, X. Zhang, J. Ma, and J. Zhang, "Design of motion controller for satellite-borne data transmission antenna," International Journal of Aerospace Engineering, vol. 2019, Article ID 6030761, 11 pages, 2019.

[10] A. Morar, "Drive system based on five-phase stepping motor under microstepping/nanostepping mode," in Procedia Technology, L. Moldovan, Ed., pp. 591-598, Elsevier Science BV, Amsterdam, 2015.

[11] A. Kapun, A. Hace, and K. Jezernik, "Identification of stepping motor parameters," in EUROCON 2007 - The International Conference on "Computer as a Tool", Warsaw, Poland, 2007.

[12] G. Baluta, "Microstepping mode for stepper motor control," in 2007 International Symposium on Signals, Circuits and Systems, Iasi, Romania, 2007.

[13] H. Tan, J. Zhang, and W. Luo, "Design of DSP based multiple stepping-motors' micro-stepping driving controller," in IEEE International Conference on Mechatronics, 2005. ICM '05, Taipei, Taiwan, 2005.

[14] W. Zhang, Structural Design Analysis and Test of a Spaceborne Sata Transmission Antenna, Xidian University, 2013.

[15] S. Wu and W. Cheng, "Two-axes mechanism for satellite antenna disturbance characteristics simulation and experiment," Journal of Beijing University of Aeronautics and Astronautics, vol. 37, no. 11, pp. 1446-1450, 2011.

[16] H. U. Oh, S.-H. Jeon, T.-H. Kim, and Y.-H. Kim, "Experimental feasibility study for micro-jitter attenuation of stepperactuated X-band antenna-pointing mechanism by using pseudoelastic SMA mesh washer," Smart Materials and Structures, vol. 24, no. 4, article 045010, 2015.

[17] H. Liu, X. Jiang, Y. Wen et al., "Analysis and verification of high pointing accuracy antenna for data transmission on GF- 
5 satellite," Aerospace Shanghai, vol. 36, Supplement 2, pp. 2429, 2019.

[18] Y. Zhou and J. Zhang, "Modeling and simulation of driving data transmission antenna," Chinese Space Science and Technology, vol. 34, no. 6, pp. 31-37, 2014.

[19] J. Song, "Vibration analysis of a data transmission antenna on high orbit satellite," Chinese Journal of Engineering Design, vol. 26, no. 3, pp. 274-279, 2019.

[20] L. Cao, Z.-C. Zhou, and Q. Guang-Ji, "Flexible coupling dynamics modeling and simulation of variable configuration spacecraft oriented control," Gongcheng Lixue/Engineering Mechanics, vol. 30, no. 8, pp. 266-271, 2013.

[21] Y. Yang, W. Cheng, S. Wu, and G. Wang, "Experiment and simulation of electromagnetic stiffness for stepper motor," Applied Mechanics and Materials, vol. 29-32, pp. 1567-1573, 2010.

[22] X. Liu, Research on the integrated modeling and analysis technologies of micro-vibration on spacecraft, Harbin Institute of Technology, 2018.

[23] J. P. Chen, W. Cheng, and M. Li, "Modeling, measurement and simulation of the disturbance torque generated via solar array drive assembly," Science China Technological Sciences, vol. 61, no. 4, pp. 587-603, 2018.

[24] M. Sattar and C. Wei, "Analysis of coupled torsional disturbance behavior of micro-stepped solar array drives," Journal of Sound and Vibration, vol. 442, pp. 572-597, 2019.

[25] J. P. Chen and W. Cheng, "Low-frequency compensation of piezoelectric micro-vibrations test platform," Tehnicki vjesnik - Technical Gazette, vol. 23, no. 5, 2016. 